\title{
Front Matter: Volume 10556
}

, "Front Matter: Volume 10556," Proc. SPIE 10556, Advances in Display Technologies VIII, 1055601 (12 June 2018); doi: 10.1117/12.2322864

SPIE. Event: SPIE OPTO, 2018, San Francisco, California, United States 


\title{
PROCEEDINGS OF SPIE
}

\section{Advances in Display Technologies VIII}

\author{
Liang-Chy Chien \\ Tae-Hoon Yoon \\ Qiong-Hua Wang \\ Editors
}

31 January-1 February 2018

San Francisco, California, United States

Sponsored and Published by

SPIE

Volume 10556 
The papers in this volume were part of the technical conference cited on the cover and title page. Papers were selected and subject to review by the editors and conference program committee. Some conference presentations may not be available for publication. Additional papers and presentation recordings may be available online in the SPIE Digital Library at SPIEDigitallibrary.org.

The papers reflect the work and thoughts of the authors and are published herein as submitted. The publisher is not responsible for the validity of the information or for any outcomes resulting from reliance thereon.

Please use the following format to cite material from these proceedings:

Author(s), "Title of Paper," in Advances in Display Technologies VIII, edited by Liang-Chy Chien, Tae-Hoon Yoon, Qiong-Hua Wang, Proceedings of SPIE Vol. 10556 (SPIE, Bellingham, WA, 2018) Seven-digit Article CID Number.

ISSN: 0277-786X

ISSN: 1996-756X (electronic)

ISBN: 9781510615977

ISBN: 9781510615984 (electronic)

Published by

SPIE

P.O. Box 10, Bellingham, Washington 98227-0010 USA

Telephone +1 3606763290 (Pacific Time) · Fax +1 3606471445

SPIE.org

Copyright (C) 2018, Society of Photo-Optical Instrumentation Engineers.

Copying of material in this book for internal or personal use, or for the internal or personal use of specific clients, beyond the fair use provisions granted by the U.S. Copyright Law is authorized by SPIE subject to payment of copying fees. The Transactional Reporting Service base fee for this volume is $\$ 18.00$ per article (or portion thereof), which should be paid directly to the Copyright Clearance Center (CCC), 222 Rosewood Drive, Danvers, MA 01923. Payment may also be made electronically through CCC Online at copyright.com. Other copying for republication, resale, advertising or promotion, or any form of systematic or multiple reproduction of any material in this book is prohibited except with permission in writing from the publisher. The CCC fee code is 0277$786 \mathrm{X} / 18 / \$ 18.00$.

Printed in the United States of America.

Publication of record for individual papers is online in the SPIE Digital Library.

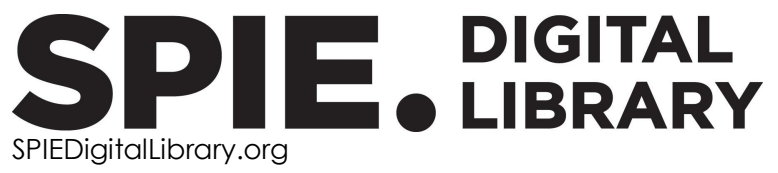

Paper Numbering: Proceedings of SPIE follow an e-First publication model. A unique citation identifier (CID) number is assigned to each article at the time of publication. Utilization of CIDs allows articles to be fully citable as soon as they are published online, and connects the same identifier to all online and print versions of the publication. SPIE uses a seven-digit CID article numbering system structured as follows:

- The first five digits correspond to the SPIE volume number.

- The last two digits indicate publication order within the volume using a Base 36 numbering system employing both numerals and letters. These two-number sets start with 00, 01, 02, 03, 04, 05, 06, 07, 08, 09, OA, OB ... 0Z, followed by 10-1Z, 20-2Z, etc. The CID Number appears on each page of the manuscript. 


\title{
Contents
}

\author{
$\checkmark \quad$ Authors \\ vii Conference Committee
}

SESSION 13 3D, LIGHT-FIELD, AND AR/VR DISPLAYS

1055603 Divers augmented vision display (DAVD) emerging technology development [10556-2]

1055604 Full-parallax three-dimensional display with spherical symmetry [10556-3]

1055605 Horizontal-parallax-only light-field display with cylindrical symmetry [10556-4]

$1055606 \quad$ Hybrid light-field display [10556-5]

SESSION 2 DISPLAY MATERIALS AND SYSTEMS

$1055607 \quad$ Fast switching of vertically aligned nematic liquid crystals by two-dimensional confinement with virtual walls (Invited Paper) [10556-6]

1055609 Negative dispersion retarder for the display compensation film (Invited Paper) [10556-8]

10556 OA Polymer-based fabrication of coupled microlens arrays for application to heads-up display [10556-9]

10556 OB End-user acceptance of anti-glare glasses [10556-10]

\section{SESSION $3 \quad$ DISPLAY MANUFACTURING AND METROLOGY}

10556 OC Color volumes in lab and ICtCp color spaces for viewing angle color characterization of QLED and OLED HDR/WCG displays (Invited Paper) [10556-1 1]

10556 OD Real-time integral imaging pickup system using camera array (Invited Paper) [10556-12]

10556 OE Stress metrology for flat-panel displays G6 and bigger [10556-13]

$10556 \mathrm{OH}$ Development of a color mixer for mixed color education and its outreach activities [10556-16] 


\section{SESSION 4 EMISSIVE DISPLAYS}

10556 Ol High-resolution active-matrix 10- $\mu \mathrm{m}$ pixel-pitch GaN LED microdisplays for augmented reality applications [10556-17]

10556 OK Light trapping for outside laser-display light harvesting [10556-20]

\section{POSTER SESSION}

$10556 \mathrm{OL} \quad$ Retinal projection type super multi-view 3D head-mounted display using the time division projection optical system [10556-22]

10556 OM A multi-object oriented iterative closest point algorithm in augmented reality [10556-23]

1055600 Partially coherent holographic display based on human factors engineering [10556-25] 


\title{
Authors
}

Numbers in the index correspond to the last two digits of the seven-digit citation identifier (CID) article numbering system used in Proceedings of SPIE. The first five digits reflect the volume number. Base 36 numbering is employed for the last two digits and indicates the order of articles within the volume. Numbers start with 00, 01, 02, 03, 04, 05, 06, 07, 08, 09, OA, OB...0Z, followed by 10-12, 20-2Z, etc.

\author{
Abdollahi, H., OA \\ Adrian, Julien, $\mathrm{OB}$ \\ Aventurier, Bernard, 0 \\ Azuma, $\mathrm{Y} ., \mathrm{OH}$ \\ Berger, Frédéric, 01 \\ Blanc, Pierre, $0 \mathrm{C}$ \\ Boher, Pierre, OC \\ Cao, Liangcai, 00 \\ Chen, J., $\mathrm{OH}$ \\ Chen, Liang, OK \\ Chen, Yu, OK \\ Choi, Geunseop, 04, 05 \\ Choi, Tae-Hoon, 07 \\ Choi, Yeongyu, 07 \\ Daami, Anis, 01 \\ Dupont, Bertrand, 0 I \\ Dupré, Ludovic, 01 \\ Gallagher, Dennis G., 03 \\ Hahn, Joonku, 04, 05 \\ $\mathrm{He}$, Zehao, 00 \\ Henry, Franck, 0 l \\ Heo, Daerak, 04 \\ Holzman, J. F., OA \\ Hue, David, OB \\ Hughes, William W., 03 \\ Jeon, Byoung-Gyu, 07 \\ Jeon, Hosung, 05 \\ Jiao, Cheng, OK \\ Jin, Guofan, 00 \\ Jin, X., OA \\ Kim, Dongyeon, 06 \\ Kim, Hwi, 05 \\ Konda, Tadayuki, OL \\ Kume, $\mathrm{Y}$., $\mathrm{OH}$ \\ Le Brun, Johan, OB \\ Lee, Byoungho, 06 \\ Lee, Ji-Hoon, 09 \\ Lee, Seungjae, 06 \\ Leroux, Thierry, OC \\ Li, Jianli, OK \\ Lim, Sungjin, 04 \\ Manley, Richard J., 03 \\ Mathieu, Lydie, ol \\ Moon, Seokil, 06 \\ Moriyama, T., $\mathrm{OH}$ \\ Nakadate, S., $\mathrm{OH}$ \\ Olivier, François, OI \\ Park, H. S., OA \\ Pilcher, Allie M., 03
}

Porte, Sophie, OB

Renet, Sébastien, 0 I

Sarrasin, Denis, 0

Shibuya, M., $\mathrm{OH}$

Song, Wen, OK

Stoeber, B., OA

Su, Ping, 00

Takahashi, Hideya, OL

Tanaka, Katsuhisa, OL

Templier, François, 0 I

Walecki, Wojtek J., OE

Wang, Guanjun, OK

Wang, Kaian, OK

Wang, Qiong-Hua, OD

Wang, Shigang, OM

Wang, Zhenhao, OM

Woo, Jae-Hyeon, 07

Xing, Yan, OD

Xiong, Zhao-Long, OD

Yan, W., OA

Yang, Jidong, OK

Yoon, Tae-Hoon, 07

Yoshimoto, Kayo, $\mathrm{OL}$

Zhang, Dao, OK

Zhang, Hao, $0 \mathrm{O}$

Zhang, Yu Ping, OK

Zhao, Min, OD

Zhao, Yan, OM 
Proc. of SPIE Vol. 10556 1055601-6

Downloaded From: https://www.spiedigitallibrary.org/conference-proceedings-of-spie on 26 Apr 2023 Terms of Use: https://www.spiedigitallibrary.org/terms-of-use 


\title{
Conference Committee
}

\author{
Symposium Chairs
}

Connie J. Chang-Hasnain, University of California, Berkeley (United States)

Graham T. Reed, Optoelectronics Research Centre, University of Southampton (United Kingdom)

Symposium Co-chairs

Jean Emmanuel Broquin, IMEP-LAHC (France)

Shibin Jiang, AdValue Photonics, Inc. (United States)

Program Track Chair

Liang-Chy Chien, Kent State University (United States)

Conference Chairs

Liang-Chy Chien, Kent State University (United States)

Tae-Hoon Yoon, Pusan National University (Korea, Republic of)

Qiong-Hua Wang, Sichuan University (China)

Conference Program Committee

Karlheinz Blankenbach, Pforzheim Universität (Germany)

Pierre M. Boher, ELDIM (France)

Liangcai Cao, Tsinghua University (China)

Cheng-Huan Chen, National Tsing Hua University (Taiwan)

Janglin Chen, Industrial Technology Research Institute (Taiwan)

Jurgen H. Daniel, PARC, A Xerox Company (United States)

Paul S. Drzaic, Apple Inc. (United States)

Mark Finn, Apple Inc. (United States)

Norbert Fruehauf, Universität Stuttgart (Germany)

Nobuyuki Hashimoto, Citizen Holdings Company, Ltd. (Japan)

Klaus Hecker, VDMA (Germany)

Alex Henzen, IRX-Innovations B.V. (Netherlands)

Yi-Pai Huang, National Chiao Tung University (Taiwan)

Lachezar Komitov, University of Gothenburg (Sweden)

Byoungho Lee, Seoul National University (Korea, Republic of)

Jiun-Haw Lee, National Taiwan University (Taiwan)

Sin-Doo Lee, Seoul National University (Korea, Republic of)

Kars-Michiel H. Lenssen, Philips Research Nederland B.V.

(Netherlands) 
Akihiro Mochizuki, i-CORE Technology, LLC (United States) Keith Rollins, DuPont Teijin Films U.K. Ltd. (United Kingdom)

Robert A. Sprague, Amazon Lab126 (United States)

Andrew J. Steckl, University of Cincinnati (United States)

Michael Wittek, Merck KGaA (Germany)

\section{Session Chairs}

1 3D, Light-Field, and AR/VR Displays

Qiong-Hua Wang, Sichuan University (China)

2 Display Materials and Systems

Akihiro Mochizuki, i-CORE Technology, LLC (United States)

3 Display Manufacturing and Metrology

Tae-Hoon Yoon, Pusan National University (Korea, Republic of)

4 Emissive Displays

Jiun-Haw Lee, National Taiwan University (Taiwan) 